\title{
Transport and Localisation in the Presence of Strong Structural and Spin Disorder
}

\author{
Sanjeev Kumar ${ }^{1}$ and Pinaki Majumdar ${ }^{2}$ \\ ${ }^{1}$ Institute for Physics, Theoretical Physics III, Electronic Correlations and Magnetism, \\ University of Augsburg, 86135 Augsburg, Germany. \\ 2 Harish-Chandra Research Institute, Chhatnag Road, Jhusi, Allahabad 211 019, India
}

(May 22, 2005)

\begin{abstract}
We study a tight binding model including both on site disorder and coupling of the electrons to randomly oriented magnetic moments. The transport properties are calculated via the Kubo-Greenwood scheme, using the exact eigenstates of the disordered system and large system size extrapolation of the low frequency optical conductivity. We first benchmark our method in the model with only structural disorder and then use it to map out the transport regimes and metal- insulator transitions in problems involving $(i)$ scattering from random magnetic moments, and $(i i)$ the combined effect of structural disorder and magnetic scattering. We completely map out the dependence of the d.c conductivity on electron density $(n)$ the structural disorder $(\Delta)$ and the magnetic coupling $\left(J^{\prime}\right)$, and locate the insulator-metal phase boundary in the space of $n-\Delta-J^{\prime}$. These results serve as a reference for understanding transport in systems ranging from magnetic semiconductors to double exchange 'colossal magnetoresistance' systems. A brief version of this study appears in our earlier paper Europhys. Lett. 65, 75 (2004).
\end{abstract}

\section{INTRODUCTION}

The most commonly studied case of localisation pertains to non interacting electrons in the background of structural disorder. There is a large body of work ${ }^{1-4}$, analytical and numerical, as well as experimental studies, that have focused on this problem. The principal qualitative result of these investigations is that in one and two dimensions all electronic eigenstates are localised for arbitrarily weak disorder, while in three dimension we need a critical disorder for complete localisation. In three dimension, at a given disorder, all states beyond an energy $\epsilon_{c}$ of the band center are localised and the system is metallic or insulating depending on whether the Fermi level, $\epsilon_{F}$, lies in the region of extended states or localised states. The 'mobility edge', $\epsilon_{c}$, collapses to the band center as the disorder is increased, driving the Anderson metal-insulator transition (MIT).

The presence of magnetic moments in a metal brings in several new effects, depending on the strength of electronspin coupling $\left(J^{\prime}\right)$, the concentration of moments $\left(n_{m a g}\right)$, the extent of disorder, and the 'character' (small or large $S)$ of the moment.

In the 'quantum limit', $2 S \sim 1$, and for antiferromagnetic coupling, the basic physics is contained in the Kondo effect. For $n_{\text {mag }} \ll 1$, the magnetic moments act as 'Kondo impurities' whose effects ${ }^{5}$ are now well understood. For $n_{\text {mag }} \sim 1$, i.e, the concentrated Kondo limit, there can be various phases depending on electronspin coupling and disorder. The ground state could be a non magnetic 'heavy Fermi liquid' 6 , or a spin glass ${ }^{7}$, or a magnetically ordered $\operatorname{state}^{8}$. The physics of these Kondo lattice, with quantum spins, is a vast area of research. In this paper, however, we will avoid the issues of heavy fermion physics and focus instead on electronspin systems involving "large $S$ ", i.e, effectively 'classical' moments.

For classical moments also, the effects vary depending on $n_{\text {mag }}$, electron density, $J^{\prime}$, and the extent of disorder. A wide variety of magnetic systems ${ }^{9-17}$ are described, to a first approximation, by electrons locally coupled to $d$ or $f$ moments, with $2 S \gg 1$, and moving in a structurally disordered background. The magnetic ground state could be ferromagnetic, or a more complicated ordered state, or a spin glass. Transport often involves insulator-metal transitions and colossal magnetoresistance. The simplest Hamiltonian capturing these effects is:

$$
H=-t \sum_{\langle i j\rangle, \sigma} c_{i \sigma}^{\dagger} c_{j \sigma}+\sum_{i \sigma}\left(\epsilon_{i}-\mu\right) n_{i \sigma}-J^{\prime} \sum_{\nu} \sigma_{\nu} . \mathbf{S}_{\nu}
$$

The $t$ are nearest neighbour hopping on a simple cubic lattice. The random on site potential, $\epsilon_{i}$, is uniformly distributed between $\pm \Delta / 2$. The sites $\mathbf{R}_{\nu}$ are a subset of the cubic lattice sites, $\mathbf{R}_{i}$, and correspond to the magnetic 'dopant' locations. Even with this simple model there are four dimensionless parameters in the problem. These are disorder $\Delta / t$, magnetic coupling $J^{\prime} S / t$, electron density $n$ (controlled by $\mu$ ), and the 'density' of moments $n_{\text {mag }}$. We will eventually study the $n_{m a g}=1$ case, but retain a more general structure right now. We absorb $S$ in our magnetic coupling $J^{\prime}$, assuming $\left|\mathbf{S}_{i}\right|=1$.

Real materials have band degeneracy and additional interactions but the basic physics of several currently interesting materials arise as limiting cases of the model above. ( $i$ ) The II-VI diluted magnetic semiconductor ${ }^{9-11}$ (DMS) $\mathrm{Ga}_{1-x} \mathrm{Mn}_{x} \mathrm{As}$, exhibiting high ferromagnetic $T_{c}$, correspond to $n_{\text {mag }} \ll 1, J^{\prime} / t \sim 1$, weak disorder, and low electron density, $n<n_{\text {mag }}$. (ii) The Eu based magnetic semiconductors ${ }^{12,13}, \mathrm{EuB}_{6}$ etc, involve $n_{\text {mag }}=1$, since every Eu atom has a moment, $J^{\prime} / t \gg 1$, low carrier density, and possibly weak disorder. ( iii) The 'colossal magnetoresistance' (CMR) manganites ${ }^{14}$, specifically 
$\mathrm{La}_{1-x} \mathrm{Sr}_{x} \mathrm{MnO}_{3}$, involve $n_{\text {mag }}=1, J^{\prime} / t \gg 1$, high electron density, and moderate 'effective disorder'. To describe the more strongly resistive manganites, the Ca doped systems, say, one requires additional electronphonon interactions. (iv) The amorphous magnetic semiconductor ${ }^{15,16}, a-\mathrm{Gd}_{x} \mathrm{Si}_{1-x}$, corresponds to $J^{\prime} / t \gg$ $1, \Delta / t \gg 1$, and $n_{\text {mag }} \sim n \sim \mathcal{O}(0.1)$. Finally, $(v)$ the traditional metallic $f$ electron magnets ${ }^{17}$, correspond to $n_{\text {mag }}=1$, and moderate to strong $J^{\prime}$.

The focus in the materials above is often on magnetism rather than localisation effects. However, many of them have rather large resistivity in the paramagnetic phase, and $a-\mathrm{GdSi}$, for example, shows a metal-insulator transition at $T=0$ itself, on lowering carrier density. Since there is no direct spin-spin interaction in these systems, the local electron-spin coupling controls both the magnetic properties and the character of the electronic state.

The intimate coupling between charge transport, localisation effects, and magnetism in these systems suggest that we need to look beyond the traditional boundaries separating 'magnetism' from transport and localisation studies. A complete study of electronic resistivity as a function of temperature, for any of the materials above, requires a solution of the magnetic problem first. Since the moments are assumed to be classical, the electrons can be imagined to move in a static background comprising the (quenched) structural disorder and annealed spin disorder. Evaluating the distribution of the annealed disorder is a non trivial problem, particularly in the strong coupling (large $J^{\prime}$ ) context that is experimentally relevant. We will touch upon this in the next section, but this paper is concerned with transport and localisation effects in the fully spin disordered phase. In this limit, we will present a comprehensive discussion of the resistivity arising from the interplay of structural disorder and 'paramagnetic' scattering, and map out the metalinsulator phase diagram in terms of electron density, disorder and magnetic coupling.

There have been some studies of electronic transport in the background of random spins and structural disorder, acting independently or together. Among these, the Anderson localisation problem itself has been extensively studied, via perturbation theory ${ }^{18}$, self-consistent schemes $^{19}$, numerical techniques ${ }^{20}$, and mapping to a field theory ${ }^{21}$. Most of the qualitative issues in this context are essentially settled. Weak magnetic scattering in a structurally disordered system has been studied ${ }^{22,23}$ in the early days of weak localisation (WL) theory to clarify the 'dephasing' effect of electron spin flip on quantum interference. In the opposite limit of strong coupling, corresponding to double exchange, localisation effects have been studied ${ }^{24}$ considering both magnetic and structural disorder.

These efforts still leave a large and interesting part of $\Delta-J^{\prime}$ space unexplored. To give a few examples, there is no discussion of the following: $(i)$ the resistivity from purely magnetic scattering, as $J^{\prime}$ rises through the perturbative regime to double exchange: this is the classic problem of paramagnetic scattering in 'clean' magnets, studied earlier at weak coupling ${ }^{25,26}$. (ii) the effect of spin disorder on the Anderson transition, i.e, how the 'anti-localising' effect of spin flip scattering, at weak disorder, evolves into an insulator-metal transition (IMT). This is an instance of Anderson transition with broken time reversal symmetry, and (iii) the wide 'middle', where the effect of neither $\Delta$ nor $J^{\prime}$ is perturbative and their contribution to the resistivity is not additive (i.e, violates Mathiessens rule). This is the regime relevant to DMS, CMR materials, and amorphous magnetic semiconductors.

The next section describes the transport calculation in detail. Following that we present results on transport, successively, in the structural disorder problem, the magnetic disorder problem, and the simultaneous effect of both. This paper follows up on our earlier short paper ${ }^{27}$.

\section{COMPUTATIONAL SCHEME}

Although we will work with random uncorrelated spins, viewing the magnetic disorder as quenched, let us highlight how the 'true' spin distribution can be evaluated, and the limit where the background can be considered random. Following that we describe our transport calculation method.

\section{A. The spin distribution}

The 'structural' variables $\epsilon_{i}$ are quenched, and have a specified distribution, but the spin orientations $\mathbf{S}_{i}$ are not known a priori. The system chooses a spin configuration, at $T=0$, to optimise the total energy. To calculate the 'true' ground state properties, or finite temperature transport, we need to solve for the spin distribution first and then evaluate electronic properties in these spin background. Denoting the full spin configuration as $\left\{\mathbf{S}_{i}\right\}$, the spin distribution $P\left\{\mathbf{S}_{i}\right\}$ is given by:

$$
\begin{aligned}
P\left\{\mathbf{S}_{i}\right\} & =Z^{-1} \operatorname{Tr} e^{-\beta H} \\
Z & =\int \mathcal{D} \mathbf{S}_{i} \operatorname{Tr} e^{-\beta H}
\end{aligned}
$$

where $Z$ is the full partition function of the system, and the 'trace' is over fermionic variables. Equivalently, the effective classical 'Hamiltonian' controlling the Boltzmann weight for spins is:

$$
H_{e f f}\left\{\mathbf{S}_{i}\right\}=-\frac{1}{\beta} \log \operatorname{Tr} e^{-\beta H}
$$

$H_{\text {eff }}$ is the fermion (free) energy in the background $\left\{\mathbf{S}_{i}\right\}$.

To make more sense of the formal expression above, consider $J^{\prime} / t \ll 1$. In this case we can expand the fermion (free) energy in powers of $J^{\prime}$. For a non disordered system this leads to the standard RKKY 
coupling $^{28}$ between the classical spins, while the presence of structural disorder, leads to a 'bond disordered' RKKY model: $H_{e f f} \sim \sum_{i j} J_{i j} \mathbf{S}_{i} . \mathbf{S}_{j}$, where the exchange $J_{i j}$ are $\sim J^{\prime 2} \chi_{i j}$ the $\chi_{i j}$ being the non local spin response function of the disordered, $J^{\prime}=0$, electron system. Having obtained the effective spin Hamiltonian, the transport properties are to be calculated by considering electron motion in the backgrounds $\left\{\epsilon_{i}, \mathbf{S}_{i}\right\}$ where the $\left\{\mathbf{S}_{i}\right\}$ are equilibrium configurations of $H_{\text {eff }}$ for a specified realisation of disorder $\left\{\epsilon_{i}\right\}$.

At strong coupling, i.e, large $J^{\prime}$, the fermion trace cannot be analytically evaluated, and it is no longer possible to write an explicit spin Hamiltonian. We need special techniques to anneal the spins. The magnetic order and the complete transport properties in such (disordered) Kondo lattice models is discussed elsewhere ${ }^{29,30}$.

The complications of the magnetic problem can be avoided if we assume a spin distribution. The simplest distribution one can assume corresponds to uncorrelated random spins. This is physically relevant in two limits.

(i) At sufficiently high temperature, compared to the magnetic ordering scales in the problem, the spins are essentially randomly fluctuating, with only short range correlation. The magnetic ordering scale for $J^{\prime} / t \ll 1$ is $\sim f_{1}(n) J^{\prime 2} / t$, while for $J^{\prime} / t \gg 1$ the ordering scale is $\sim f_{2}(n) t$, where $f_{1}$ and $f_{2}$ are electron density dependent dimensionless coefficients and $f_{2}^{\max } \sim 0.1$. Compared to the typical Fermi energy, $\sim z t$, where $z$ is the coordination number of the lattice, these scales are all small. We use a $T=0$ formulation for transport, i.e, we do not use Fermi factors, but given the smallness of $T_{c} / \epsilon_{F}$, our results would be relevant even in the 'real' paramagnetic phase. (ii) Another situation in which a random spin configuration is appropriate is a 'spin glass', likely to occur in strongly disordered systems ${ }^{31}$. There are always short range correlations in a spin glass but if we ignore their effect on transport then at all temperature the transport in such a system can be described, approximately, in terms of a random spin background.

\section{B. Conductivity calculation}

In the linear response regime, the Kubo formula can be used to calculate the conductivity of a system. The general expression ${ }^{32}$, involving matrix elements between many body states, simplifies significantly for noninteracting systems. This 'Kubo-Greenwood' result can be computed purely in terms of single particle eigenfunctions and energies.

The numerical difficulty with this result lies in implementing it for a finite size system, where the spectrum is discrete, with gaps $\mathcal{O}(1 / N)$, with $N$ being the number of sites in the system. Since the 'd.c' conductivity involves transitions between essentially degenerate states at $\epsilon_{F}$, it cannot be calculated with control on small systems. As a result, instead of computing the 'Kubo conductivity' it is more usual to compute the 'Landauer conductance', $G$, of a finite system coupled to leads ${ }^{33}$, and infer the conductivity from the length dependence of $G$.

We pursue the Kubo approach, indirectly, through a calculation of the low frequency optical conductivity for a $L_{T} \times L_{T} \times L$ geometry. The advantage of calculating the conductivity this way is, $(i)$ it ties in with diagonalisation that one may have to do for the magnetic problem, and $(i i)$ it allows access to the optical conductivity, without added effort, and can reveal the significantly non Drude nature of $\sigma(\omega)$ at strong disorder. The principal disadvantage is, this scheme cannot be pushed beyond $N \sim 10^{3}-10^{4}$, and is therefore not useful for accessing critical properties.

For disordered non interacting systems, the Kubo formula, at $T=0$, is:

$$
\sigma(\omega)=\frac{A}{N} \sum_{\alpha, \beta}\left(n_{\alpha}-n_{\beta}\right) \frac{\left|f_{\alpha \beta}\right|^{2}}{\epsilon_{\beta}-\epsilon_{\alpha}} \delta\left(\omega-\left(\epsilon_{\beta}-\epsilon_{\alpha}\right)\right)
$$

with $A=\pi e^{2} / \hbar a_{0}, a_{0}$ being the lattice spacing, and $n_{\alpha}=$ $\theta\left(\mu-\epsilon_{\alpha}\right)$. The $f_{\alpha \beta}$ are matrix elements of the current operator $j_{x}=i t \sum_{i, \sigma}\left(c_{i+x a_{0}, \sigma}^{\dagger} c_{i, \sigma}-h . c\right)$, between exact single particle eigenstates $\left|\psi_{\alpha}\right\rangle,\left|\psi_{\beta}\right\rangle$, etc, and $\epsilon_{\alpha}, \epsilon_{\beta}$ are the corresponding eigenvalues.

The conductivity above is prior to disorder averaging. Notice that the $\delta$ function constraint cannot be satisfied for arbitrary frequency in a finite system. So we can neither calculate the d.c conductivity, $\sigma_{d c}$, directly, nor estimate $\sigma(\omega)$ at some arbitrary externally specified frequency. However, we can still calculate the 'average' conductivity over a frequency interval $\Delta \omega$, defined below, and we use the following strategy to extract $\sigma_{d c}$.

(i) The average of $\sigma(\omega)$ over the interval $[0, \Delta \omega]$ is defined as

$$
\sigma_{a v}(\Delta \omega, \mu, N)=\frac{1}{\Delta \omega} \int_{0}^{\Delta \omega} \sigma(\omega, \mu, N) d \omega
$$

$\Delta \omega$ can be set independent of $N$, but we will relate them by fixing: $\Delta \omega=B / N$. We fix $B$ by setting $\Delta \omega=0.04$ for $N=1000$. The mean finite size gap is $12 / 1000 \sim 0.01$, in $3 \mathrm{~d}$, much smaller than $\Delta \omega$.

(ii) $\sigma_{a v}$ is averaged over $N_{r}$ realisations of disorder, to generate $\bar{\sigma}_{a v}(\Delta \omega, \mu, L)$. The 'noise' in $\bar{\sigma}_{a v}(\Delta \omega, \mu, L)$ falls slowly, as $1 / \sqrt{N_{r}}$. We use $N_{r} \sim 100$ for the largest sizes, to keep the computation reasonable, and use a filter to smooth the data over a small window in $\mu$.

(iii) We study the $\bar{\sigma}_{a v}(\Delta \omega, \mu, L)$ for $L_{T}=6$ and the sequence $L=24$ to $L=64$ in increments of 8 and extrapolate, $\sigma_{\text {calc }}(\mu)=\lim _{L \rightarrow \infty} \bar{\sigma}_{a v}(\Delta \omega, \mu, L)$. As specified before, $\Delta \omega=B / N$.

To calculate the full, disorder averaged, optical conductivity we use the inversion: $\sigma(\omega)=\bar{\sigma}_{a v}(\omega)+\omega \frac{d \bar{\sigma}_{a v}}{d \omega}$. The $\sigma(\omega)$ results in this paper are mostly based on a $6 \times 6 \times 32$ geometry. 


\section{TRANSPORT IN THE ANDERSON MODEL}

The metal-insulator phase boundary and the critical properties near the transition have been extensively studied $^{18-21}$ in the Anderson model. However, the actual resistivity seems to have received much less attention. As recently pointed out by Nikolic and Allen ${ }^{34}$, there is a wide regime in $\Delta$, between the Born-Boltzmann end and the scaling regime, where there are no analytic theories of transport. We study this 'old problem' in some detail because the wealth of existing results provides a benchmark for our method. There are very few exact results with which we will be able to compare our data in the magnetic scattering problems.

\section{A. Global features}

The 'global features' of transport and localisation in the Anderson model are contained in Figs.1-3. The data is obtained via the extrapolation procedure described earlier. Fig.1.(a) highlights the suppression in conductivity with increasing disorder, across the entire band. The 'weakest' disorder in this case, $\Delta=4$, is probably already outside the semiclassical Boltzmann regime. A naive $\Delta^{2}$ scaling of the resistivity still works, at the band center, between $\Delta=4$ and $\Delta=8$, but the same extrapolated to $\Delta=16$, would imply $\rho(16) / \rho(4) \sim 16$, while the ratio is actually $\sim 10^{3}$. This figure captures the expected crossover from moderate scattering, roughly following Boltzmann scaling, to localisation as $\Delta \rightarrow \Delta_{c} \sim 16.5$, the critical disorder ${ }^{35}$ at the band center. It also provides a glimpse of how the 'mobility edge' moves with increasing disorder, better quantified in Fig.3. Note that for data at a specified system size, $L=16,32$ etc, shown later, the notion of a 'mobility edge' does not make sense, and all we observe is a crossover from small to large conductivity as $\mu$ is varied. The change in $\sigma(\mu, L)$ with $L$, and the $L \rightarrow \infty$ extrapolation, is crucial for identifying the mobility edge.

The DOS plot, Fig.1.(b), illustrates the band broadening, and in Fig.3.(b) we have compared our band edge energy with earlier CPA results ${ }^{36}$. The (algebraic) average DOS is featureless and non critical and does not play an interesting role in the problem.

Since the band broadens significantly with disorder, $\sigma(\mu)$ by itself does not provide the density dependence of the conductivity. Fig.2 takes into account the shift in $\mu$ required to maintain constant density (with increasing disorder) and shows $\sigma(n)$. Our density is defined as average number of electrons per site, so $n_{\max }=2$. Since the model is particle-hole symmetric we show only the regime $n=[0,1]$.

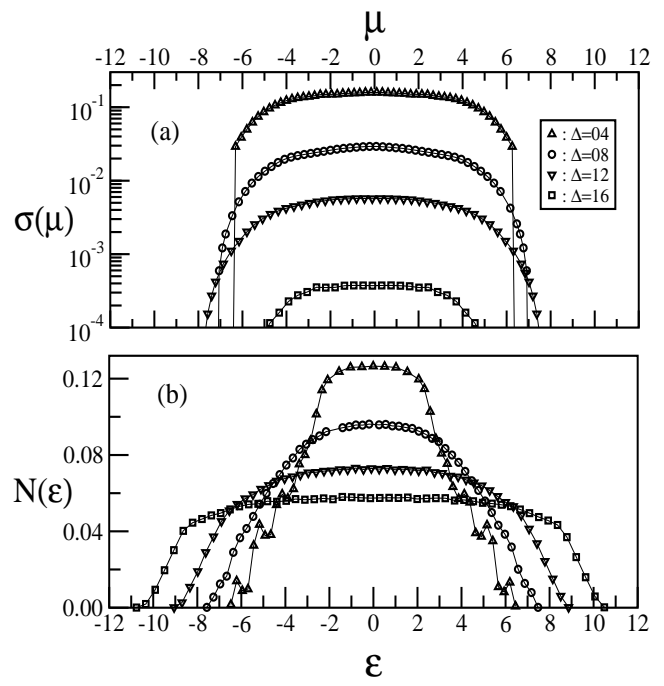

FIG. 1. Panel $(a)$. Variation of conductivity with $\mu$, and panel $(b)$. density of states, for several values of $\Delta$.

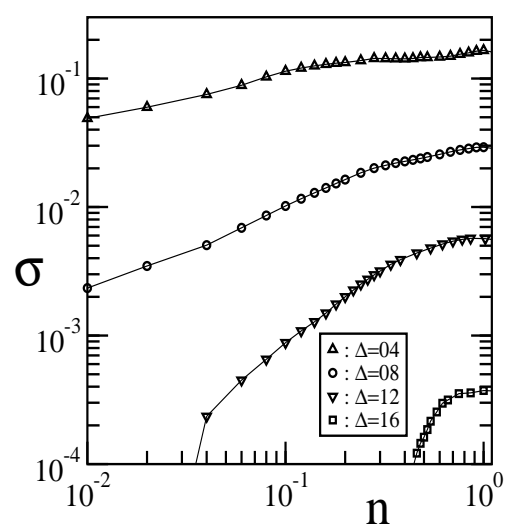

FIG. 2. Variation of conductivity with carrier density, for several $\Delta$, constructed from the $\sigma(\mu)$ and $N(\epsilon)$ data in Fig.1

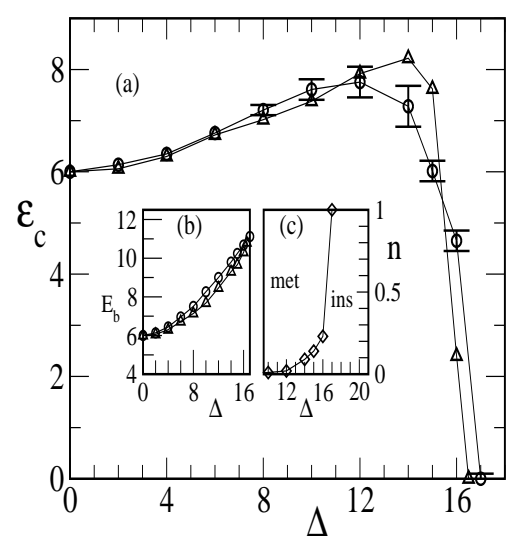

FIG. 3. Main panel, $(a)$, shows the variation in mobility edge with disorder. We compare our results, circles, with earlier work ${ }^{36}$, triangles. Inset $(b)$ shows the 'band edge', and (c) the fraction of localised states at large $\Delta$. 
To get a feel for the magnitude of the conductivity, which we measure in units of $\pi e^{2} /\left(\hbar a_{0}\right)$, note that the Mott 'minimum metallic conductivity', $\sigma_{M o t t}$, at the band center ${ }^{4}$, is roughly $\sim 0.03 e^{2} /\left(\hbar a_{0}\right)$. Our dimensionless conductivity $\sigma_{\text {calc }}$, shown in the figures, can be converted to real units, $\sigma_{\text {actual }}$, by using

$$
\sigma_{\text {actual }} \sim 100 * \sigma_{M o t t} * \sigma_{\text {calc }}
$$

where we use $\sigma_{M o t t}=0.03 e^{2} /\left(\hbar a_{0}\right)$. The results we show in the present spin degenerate problem includes a factor of 2 to account for the two spin channels. This is important to compare with the magnetic scattering problems later. The conductivity per spin channel falls below $\approx 10^{-2}$ for $\Delta \gtrsim 8$. This implies that beyond $\Delta \approx 8$, $\sigma<\sigma_{\text {Mott }}$ in the Anderson model.

The main panel in Fig. 3 shows the variation in mobility edge with increasing disorder. Our error estimates are based on the shift in $\epsilon_{c}$ as we change from moderate to zero filtering of the $\bar{\sigma}(\mu, L)$ data. We show some earlier standard result ${ }^{36}$ for comparison. The best current result on $\Delta_{c}$ is 16.5 , our method yields $\Delta_{c} \sim 17$. Our results on the band edge, Fig.3.(b), match reasonably with earlier CPA based results. Note that while the mobility edge has a 're-entrant' behaviour, the fraction of localised states in the band, Fig.3.(c), increases monotonically with disorder.

\section{B. Transport regimes}

There are tentatively three transport regimes in the Anderson model. These are $(i)$ the perturbative Born scattering regime, described by the Boltzmann transport equation and the low order corrections in $\left(k_{F} l\right)^{-1}$. This corresponds to $\Delta / W \ll 1$, where $W=12 t$. ( $i i)$ The wide intermediate coupling regime $\Delta / W \sim \mathcal{O}(1)$, and (iii) the 'scaling' region, $\Delta \rightarrow \Delta_{c}$, near the MIT.

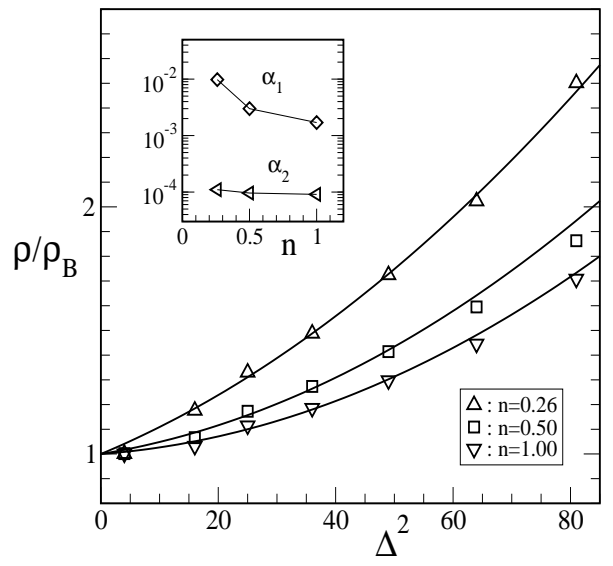

FIG. 4. Variation of the resistivity, normalised to the Born resistivity, with disorder. The firm lines are fits of the form $1+\alpha_{1}(n) \Delta^{2}+\alpha_{2}(n) \Delta^{4}$. Inset shows the coefficients $\alpha_{1}, \alpha_{2}$.
We analyse the data, with increasing $\Delta$, in the sequence $(i) \rightarrow($ ii $) \rightarrow($ iii $)$.

\section{Perturbative regime}

To leading order, the scattering rate from the disorder is $\tau_{\Delta}^{-1} \sim 2 \pi N\left(\epsilon_{F}\right)\left\langle\epsilon_{i}^{2}\right\rangle$. The second moment of the random potential is, $\left\langle\epsilon_{i}^{2}\right\rangle \sim \Delta^{2} / 12$. Since $N\left(\epsilon_{F}\right) \sim 0.13$, at the band center, Fig.1.(b), the scattering rate, $\Gamma_{\Delta}=$ $\tau_{\Delta}^{-1} \approx \Delta^{2} /(15 t)$

The three related quantities which define Boltzmann transport are $(i)$ the scattering rate, $\Gamma_{\Delta}$, defined above, (ii) the (inverse) mean free path $a_{0} / l \sim 0.03(\Delta / t)^{2}$ at the band center, and (iii) the Born-Boltzmann conductivity $\sigma_{B} \approx 1.62\left(\pi e^{2} / \hbar a_{0}\right) *(t / \Delta)^{2}$ per spin channel, again at the band center. In addition the optical conductivity should have the Drude form $\sigma(\omega)=\sigma_{B} /\left(1+\left(\omega^{2} / \Gamma_{\Delta}^{2}\right)\right)$, and the 'width' in the optical conductivity can be checked against the magnitude of d.c conductivity.

Using the form for $\sigma_{B}$, the conductivity at $\Delta=4$, assuming Boltzmann transport, would be approximately $0.1\left(\pi e^{2} / \hbar a_{0}\right)$ per spin channel, i.e $\sim 0.2$ in our units including spin degeneracy. Our data, Fig.2, gives a value $\sim 0.19$ at the band center. The crude Boltzmann scaling is obvious from the moderate $\Delta$ results in Fig. 1 and Fig.2. In Fig. 4 we attempt to quantify the corrections to the Boltzmann result, still staying far from the localisation regime.

The weak localisation corrections that arise beyond Boltzmann transport control the resistivity in one and two dimension. These are quantum interference effects, formally arising from the 'Cooperon' corrections. A similar argument would lead us to believe that in three dimension $^{1}$ the leading correction beyond the Boltzmann results should be $\delta \sigma \propto-\left(k_{F} l\right)^{-1}$. Since $\left(k_{F} l\right)^{-1} \propto \Delta^{2}$ and the Boltzmann conductivity $\sigma_{B} \propto k_{F} l$, the net conductivity would be expected to have the form $\sigma \sim$ $\sigma_{B}\left(1-\mathcal{O}\left(\left(k_{F} l\right)^{-2}\right)\right)$, i.e, $\sigma(\Delta) \sim \Delta^{-2}\left(1-\mathcal{O}\left(\Delta^{4}\right)\right)$. In that case, the resistivity should have a form $\rho(\Delta) \sim$ $\rho_{B}(\Delta)\left(1+\mathcal{O}\left(\Delta^{4}\right)\right)$.

Fig.4 shows $\rho(\Delta) / \rho_{B}(\Delta)$ plotted against $\Delta^{2}$ for three densities. We avoid too low a density to keep the scales comparable. The data are fitted to $\rho / \rho_{B}=1+\alpha_{1}(n) \Delta^{2}+$ $\alpha_{2}(n) \Delta^{4}$, upto $\Delta^{2}=49$ and then extrapolated to $\Delta^{2}=$ 81 .

There are two notable features: $(i)$ There is clearly a non zero coefficient $\alpha_{1}(n)$ so the equivalent of the WL corrections do not control the leading correction to $\sigma_{B}$ in three dimension. The coefficients $\alpha_{1}$ and $\alpha_{2}$ are shown in the inset in Fig.4. ( $i i)$ The 'low $\Delta$ ' fit seems to work reasonably for $\Delta \lesssim 8$, in the sense that $\rho / \rho_{B} \lesssim 2$. This qualitative correspondence with the Boltzmann result, even in the regime $a_{0} / l \gtrsim 1$, has been noticed recently ${ }^{34}$.

The first issue has been explored in detail ${ }^{37}$ by Belitz and Kirkpatrick who argue that the standard WL processes do not exhaust the leading corrections to $\sigma_{B}$ in 
three dimension. According to them, the perturbative expansion for $\sigma$, in a continuum model, has the form

$$
\sigma \sim \sigma_{B}\left\{1-a\left(k_{F} l\right)^{-1}-b\left(k_{F} l\right)^{-2} \log \left(k_{F} l\right)+\mathcal{O}\left(\left(k_{F} l\right)^{-2}\right)\right\}
$$

where $a$ and $b$ are numerical coefficients $\mathcal{O}(1)$. The WL argument would put $a=0, b=0$.

This form for the correction beyond Boltzmann transport has apparently been observed for electron mobility in dense neutral gases. The detailed coefficients in this expression would change in a tight binding model, but the key result about $k_{F} l$ dependence should survive.

\section{Intermediate coupling}

The Boltzmann result alongwith the perturabtive quantum corrections is reasonable probably upto $\Delta / W \sim$ $0.2-0.3$, although numerically the fit, in the last section, seems to describe the resistivity even upto $\Delta / W \sim 0.75$. The scaling regime, where localisation effects become visible, occurs within about $10 \%$ of $\Delta_{c}$.

Despite the correspondence of our numerical results with an extrapolation of weak coupling theory, there is no analytic framework for calculating the resistivity when the "small parameter" $\left(k_{F} l\right)^{-1} \sim a_{0} / l$ becomes $\mathcal{O}(1)$.

The paramter $a_{0} / l$ is $\mathcal{O}(1)$ for $\Delta / W \sim 0.5$ but the deviation from the Boltzmann result (at the band center) is only about $25 \%$. This has been pointed out recently by Nikolic and Allen ${ }^{34}$ and probably arises from a fortuitious cancellation of higher order corrections. The selfconsistent theory (SCT) of Vollhardt and Wolfle ${ }^{19}$ serves as an interpolating approximation in this regime.
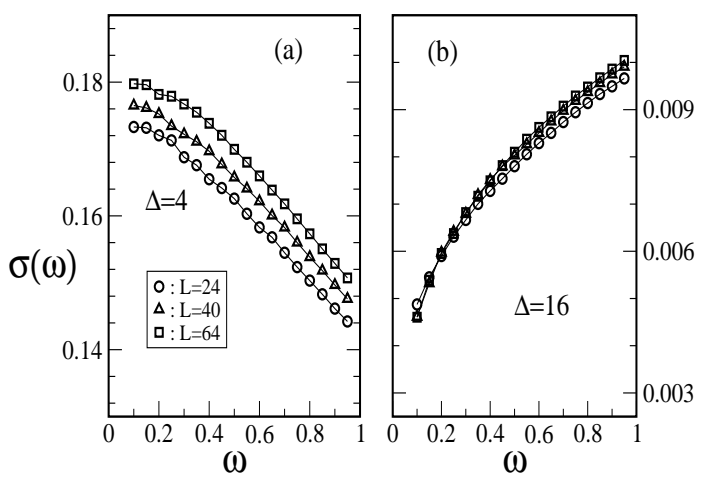

FIG. 5. The optical conductivity, at band center, for different $L$ and $\Delta$. Panel $(a)$. corresponds to the moderate disorder regime, with a Drude form for $\sigma(\omega)$, while $(b)$. is for a system on the verge of localisation (vanishing $\sigma_{d c}$ ).

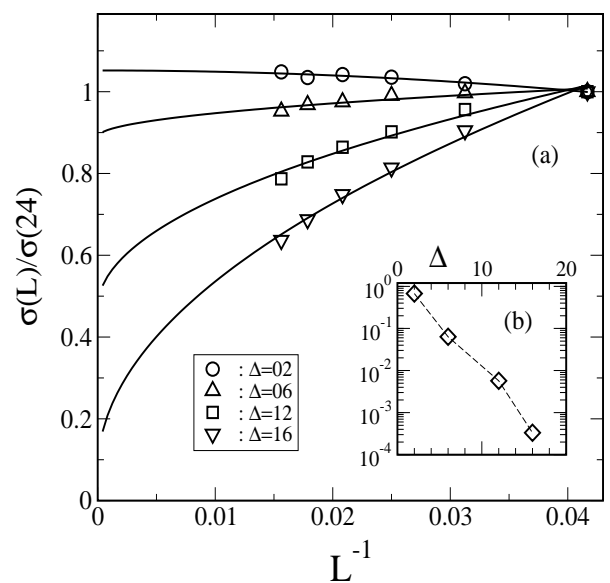

FIG. 6. The approach to the d.c conductivity, with increasing $L$ in the $L_{T} \times L_{T} \times L$ geometry. $L_{T}=6$ and the disorder is increased from the perturbative end towards localisation. The chemical potential $\mu=0$, so $n=1 . \bar{\sigma}(L)$, defined earlier in the text, is the average of $\sigma(\omega)$ over the interval $[0, \Delta \omega]$, with $\Delta \omega=1.1 / L$, at $L_{T}=6$. Inset: variation of $\bar{\sigma}(L=24)$ with $\Delta$, to illustrate the rapid fall in the reference conductivity with increasing disorder.

Within the SCT also, the conductivity at band center remains within $20 \%$ of $\sigma_{B}$ for $\Delta \lesssim 8$. The deviation from the Boltzmann result grows as we move from the band center to the band edge as evident in Fig.4.

\section{Scaling regime}

The scaling regime occurs close to critical disorder, within about $10 \%$ of $\Delta_{c}$. The conductivity in this regime varies as $\Delta_{c}-\Delta$. This regime has been extensively studied to clarify the critical properties (see, e.g. ${ }^{19}$ and references therein). We have not used a dense enough sampling in $\Delta$ for discussing the critical behaviour, and our system sizes too are not large enough for high accuracy calculation of the conductivity in this regime. However, based on results at $\Delta=16$ and $\Delta=17$ we can bracket the critical point, as shown in Fig.3.

\section{Optical conductivity}

The optical conductivity $\sigma(\omega)$ is of intrinsic interest ${ }^{38}$ and also plays a role in our method of determining the d.c conductivity. There are some exact results known on the form of the low frequency $\sigma(\omega)$ in the Anderson model.

(i) At weak disorder, when Boltzmann transport holds, the optical conductivity has the Drude form, $\sigma(\omega) \sim$ $\sigma(0) /\left(1+\omega^{2} \tau^{2}\right)$, where $\tau^{-1} \propto \Delta^{2}$ as we already know. For $\omega \tau \ll 1$ this would give us $\sigma(\omega) \sim \sigma(0)\left(1-\omega^{2} \tau^{2}\right)$. (ii) When the quantum corrections to the d.c conductivity become important the frequency dependence also picks up a non Drude form. In the intermediate disorder regime, one expects $\sigma(\omega) \sim \sigma(0)+\mathcal{O}(\Gamma \sqrt{\omega / \Gamma})$, where 
$\sigma(0)$ already incorporates corrections beyond the Boltzmann result. In this regime the conductivity rises with increasing frequency, for frequencies $\omega \ll \Gamma$. (iii) At the critical point, where the zero frequency conductivity vanishes, $\sigma(\omega) \sim \omega^{1 / 3}$, and in the localised regime $\sigma(\omega) \sim \omega^{2}$.

These results originally obtained through different techniques can be obtained in a unified way via the selfconsistent theory of Vollhardt and Wolfle.

Fig.5 demonstrates the changing character of $\sigma(\omega)$, at $n=1$, as we move from the Boltzmann regime $(\Delta=$ $2)$, to strong disorder $(\Delta=16)$. We show the data for three system sizes at each $\Delta$ to illustrate the explicit $L$ dependence in $\sigma(\omega, L)$. This is important for analysing the extrapolation needed for $\sigma_{d c}$.

There are two effects of changing system size: $(i)$ the $\sigma(\omega)$ profile itself can change with evolving system size, over the frequency range of interest, and ( $i i)$ larger system size allows access to (more dependable) low frequency data.

Fig.5.(a), the weak disorder case, reveals that the $\sigma(\omega)$ profile changes perceptibly with increasing $L$, the changes being $\mathcal{O}(5 \%)$. This implies that in our attempt to access d.c conductivity, the contribution arises not only from lowering the frequency cutoff but also moderate changes in the $\sigma(\omega)$ profile. At strong disorder, Fig.5.(b), the profile itself does not change significantly with $L$ and the key change in the $\sigma_{d c}$ estimate comes from our ability to access lower frequencies.

\section{Large $L$ extrapolation}

How important is the large $L$ extrapolation to access the d.c conductivity, i.e, what is the error if we treat the

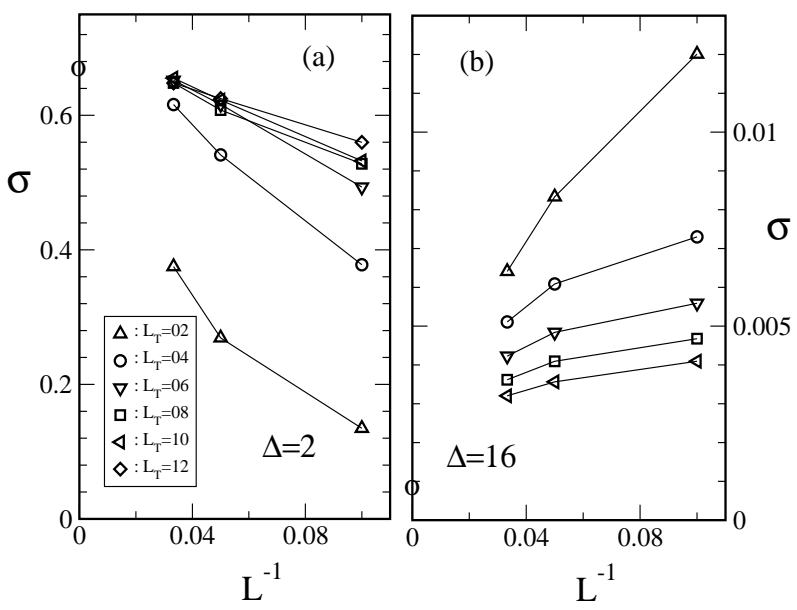

FIG. 7. Effect of transverse dimension on the large $L$ extrapolation. Carrier density $n=1$. The conductivity obtained by extrapolating the $6 \times 6 \times L$ results are shown as circles on the $y$ axes. low frequency average $\bar{\sigma}_{a v}(L)$, at some size $L$, as the bulk d.c conductivity? Fig.6 illuatrates the extrapolation based on the sequence $\{L: 24,32,40,48,56,64\}$, at $\mu=$ 0 , moving from weak to strong disorder.

In the weak disorder regime, the optical conductivity is 'flat' for $\omega \ll \tau^{-1}$ so if low enough frequencies can be accessed (given the finite size gaps), the d.c conductivity can be reasonably approximated. This is the feature observed at $\Delta=2$ in Fig.6. However, in the WL region and beyond, $\sigma(\omega)$ has non trivial frequency dependence at low $\omega$, as evident in Fig.5.(b). The corresponding low frequency average has significant $L$ dependence. Since $\sigma(\omega) \sim \sigma(0)+\mathcal{O}(\sqrt{\omega})$, the low frequency average $\sigma_{a v}(L) \sim \sigma(\infty)+\mathcal{O}(1 / \sqrt{L})$. The data at $\Delta=12,16$ show a reasonable fit to the square root form. The much stronger frequency dependence in the strong disorder regime makes a size dependent study imperative. We provide a discussion of the extrapolation scheme in an appendix.

These results illustrate the work involved in accessing the d.c conductivity, particularly in the regime of strong disorder, where a small $L$ calculation (at $L=16$ say) might overestimate the conductivity by a factor of 4 . This discrepancy worsens as $\Delta \rightarrow \Delta_{c}$ and a systematic study of size dependence is vitally important.

\section{E. Effect of the transverse dimension}

All the results quoted till now have been obtained via extrapolation on a $6 \times 6 \times L$ geometry. The $6^{2}$ cross section was chosen to allow large $L$ to be accessed. However, it is important to quantify the error involved in chosing a specific transverse dimension $L_{T}$. To this end we studied the low frequency average $\sigma_{a v}$ in a sequence $L_{T} \times L_{T} \times L$ with $L_{T}=2,4,6,8,10,12$ and $L=10,20,30$, for $\Delta=2,10$ and 16 , and $n=1$. The averaging interval $\Delta \omega$ was scaled as $1 /\left(L^{2} L_{T}\right)$ in all geometries.

Fig. 7 shows $\sigma_{a v}$ with respect to $L^{-1}$ for the sequence $L_{T}$ specified above. Panel $(a)$. shows the weak disorder, $\Delta=2$ result. Beyond $L_{T}=4$ all the curves seem to converge to $\sigma \sim 0.70$ for $L \rightarrow \infty$. The extrapolation from $L_{T}=6$, obtained using $L$ upto 64 , is shown as a circle on the $y$ axis, and is $\sim 0.68$.

For the strong disorder case, Fig.7.(b), the extrapolation for $L_{T}=6$ is shown to be $\sim 0.001$, while the large $L_{T}$ data, using $L$ upto 30 , suggests that the asymptotic value could be larger, $\sim 0.002$. This suggests that 'small' $L_{T}$ somewhat underestimates the conductivity (remember $L_{T}=1$ is one dimensional, so completely localised), while finite $L$ overestimates the conductivity. Except very close to the MIT these errors are small for the sizes we use and, as verified by the phase diagram, even the critical point is located to within $5 \%$. 


\section{SCATTERING FROM MAGNETIC DISORDER}

\section{A. Global features}

The effect of weak magnetic scattering on transport is quite similar to that of potential scattering. The effect is contained in the Born scattering rate, $\tau_{s}^{-1} \propto$ $N\left(\epsilon_{F}\right) J^{\prime 2} S^{2}$, and the weak coupling resistivity $\rho\left(J^{\prime}, n\right)$ varies as $\sim b_{1}(n) J^{\prime 2}$, where $b_{1}(n)$ is a density dependent coefficient. However, even at moderate coupling, $J^{\prime} \sim 2$, new effects begin to show up in $\sigma(\mu)$. The conductivity at half-filling, $n \sim 1$, gets suppressed more quickly than would be guessed based on the Born argument. This deviation, and its evolution with increasing $J^{\prime}$, arises from a fundamental difference between potential scattering and magnetic scattering on a 'Kondo lattice'.

There are in fact two main differences that show up beyond weak coupling. These are visible when we compare Figs.8-10, with Figs.1-4. (i) The conductivity in the potential scattering case decreases monotonically (at fixed $n$ ) with increase in disorder, vanishing at $\Delta_{c}(n)$, while in the magnetic scattering case, at a generic density, the conductivity is finite even as $J^{\prime} \rightarrow \infty$. The resistivity 'saturates' and there is no metal-insulator transition with increasing $J^{\prime}$, except in a narrow density window. (ii) The band center, $n \sim 1$, is of no particular significance in the Anderson problem, except $\Delta_{c}$ being largest. In the $J^{\prime}$ problem the response for $n \sim 1$ is dramatically different from that in the rest of the band. There is an MIT at $J^{\prime} \approx 5$. These differences can be understood from an analysis of the strong coupling end.

For $J^{\prime} / t \gg 1$ it is useful to choose a local quantisation axis at each site, for the electrons, parallel to the orientation of the spin $\mathbf{S}_{i}$. The coupling $J^{\prime} \mathbf{S}_{i}$ acts as a strong local Zeeman field on the electron. Suppose the hopping term were absent. The two local eigenfunctions at each site would have spin projections parallel and antiparallel to $J^{\prime} \mathbf{S}_{i}$, with energy $\mp J^{\prime} / 2$ respectively. The zero hopping problem leads to $N$ fold degenerate levels at $\pm J^{\prime} / 2$. The 'gap' $J^{\prime}$ plays a key role at strong coupling. The presence of hopping generates a degenerate perturbation on the locally aligned states (say), and the electrons can now 'hop' with an amplitude that depends on the orientation of nearest neighbour spins. This mechanism has been extensively discussed in the context of the double exchange model.

The mixing introduced by 'hopping' broadens the two levels into bands. For $J^{\prime} \gtrsim W / 2$ the broadening due to $t$ does not fill the gap, and the system is insulating at $n=$ 1 . For $J^{\prime}$ below this critical value, $J_{c}$, say, the DOS at band center is suppressed but finite, and the resistivity is still very large. In summary, the strong coupling physics of (incipient) band splitting controls the resistivity close to band center, and creates an essential difference, in terms of $J^{\prime}$ and $n$, with respect to standard Anderson localisation.

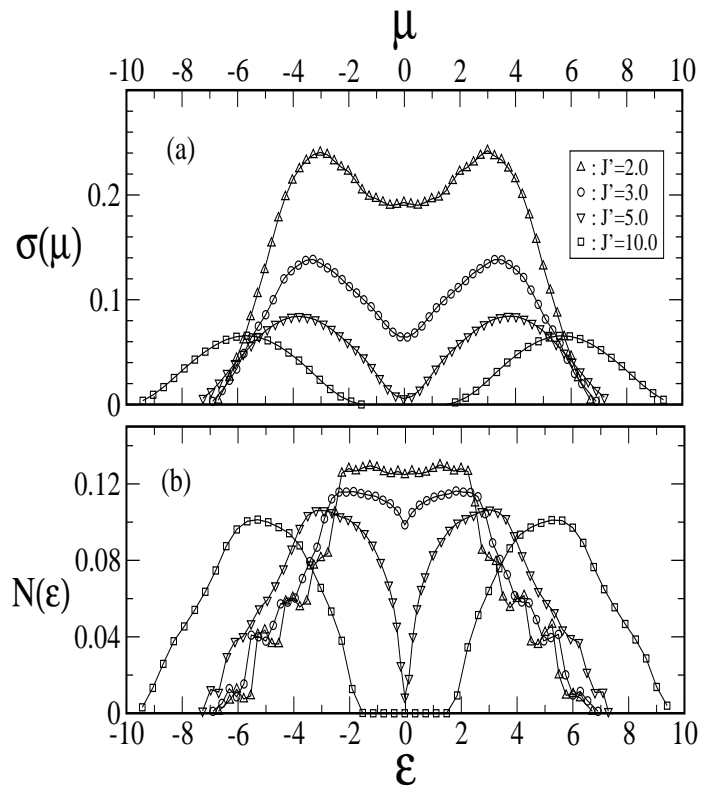

FIG. 8. (a). Conductivity as a function of Fermi energy, (b). density of states, for different values of $J^{\prime}$, in the case of pure magnetic scattering.

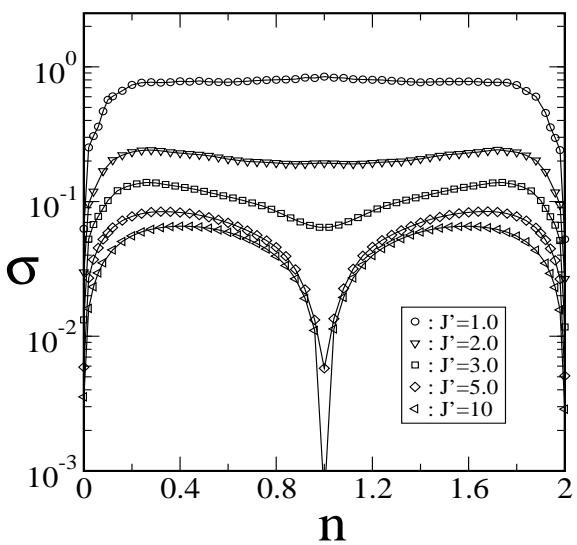

FIG. 9. Dependence of conductivity on carrier density for varying $J^{\prime}$. The conductivity scale is logarithmic.

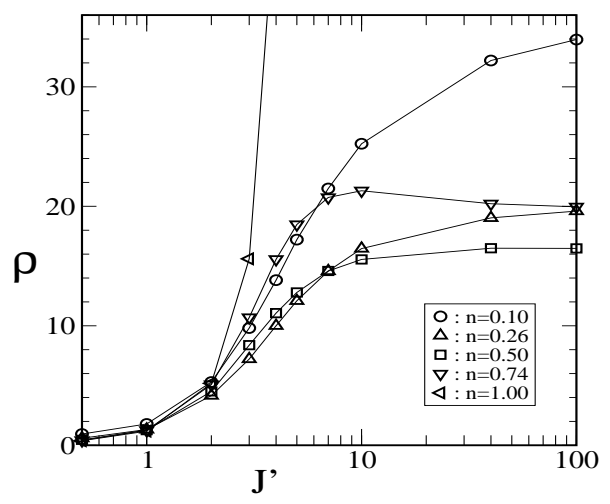

FIG. 10. Resistivity variation with $J^{\prime}$, for several electron density, from the perturbative limit to double exchange 
The saturation in $\rho\left(J^{\prime}\right)$ with increasing $J^{\prime}$, over most of the band, occurs because the effect of large $J^{\prime}$ is absorbed mainly in the band splitting. The effective disorder seen by the electrons comes from fluctuations in the hopping amplitudes, explained in the next section, and these are $\mathcal{O}(t)$. The ratio of fluctuation to mean hopping is moderate, so the large $J^{\prime}$ limit leads to a 'dirty metal' but no metal-insulator transition. This is unlike the Anderson problem where the electrons scatter off potential fluctuations whose amplitude grows with increasing $\Delta$.

From the data in Figs.8-10 we can now identify the different transport regimes.

\section{B. Transport regimes}

\section{Weak coupling: $J^{\prime} / W \ll 1$}

The magnetic scattering rate $\Gamma_{s}$ is proportional to $N\left(\epsilon_{F}\right) J^{\prime 2}$, and the weak coupling resistivity should be expandable in $\Gamma_{s}$. The lowest order term is well known, corresponding to Born scattering, with $\rho\left(J^{\prime}, n\right) \sim b_{1}(n) J^{\prime 2}$. The density dependence is similar to that for potential scattering. Assuming $\rho\left(J^{\prime}\right)$ to be analytic in $\Gamma_{s}$, i.e ignoring possible log corrections etc, Fig.11 shows a fit of the form $\rho\left(J^{\prime}, n\right) \sim b_{1}(n) J^{\prime 2}+b_{2}(n) J^{\prime 4}$ to the low $J^{\prime}$ resistivity. The $J^{\prime 2}$ character dominates upto $J^{\prime} \sim 2$, as one can see also in the $\sigma(n)$ plot in Fig.9, beyond which the quartic term becomes important. We do not know if the coefficient of the quartic term has been analytically calculated, but the sign of this term is crucial, and is density dependent, as we discuss next.

\section{Intermediate coupling: $J^{\prime} / W \sim \mathcal{O}(1)$}

As is obvious from the data in Fig.9-10, the resistivity saturates with increasing $J^{\prime}$, over most of the band. The

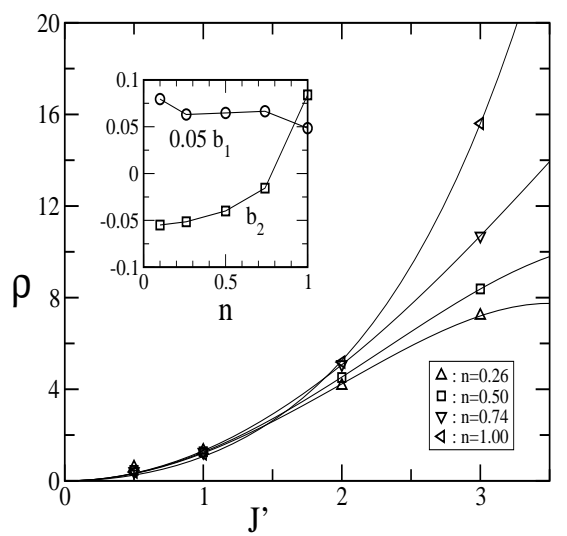

FIG. 11. Fit to the weak coupling resistivity of the form $\rho\left(J^{\prime}, n\right) \sim b_{1}(n) J^{\prime 2}+b_{2}(n) J^{\prime 4}$. The symbols are actual data and the firm lines are fits. The inset shows the $n$ dependence of the coefficients $b_{1}$ and $b_{2}$. Note the sign change in $b_{2}$. exception is the vicinity of $n=1$, and the lower edge of the band. This suggests that the correction to the Born resistivity is negative for $n$ away from $n=1$, and changes sign as $n \rightarrow 1$. Fitting the data to $\rho\left(J^{\prime}, n\right)=$ $b_{1}(n) J^{\prime 2}+b_{2}(n) J^{\prime 4}$, the coefficient $b_{2}(n)$ illustrates the crossover from saturation to escalation, as we move across the band. Fig.11 shows the fit to this form and the coefficients are shown in the inset. The 'Born' coefficient is positive throughout the band, without significant density dependence in the density interval shown. The quartic coefficient changes sign, from positive to negative, as $n$ is lowered from 1.0 to 0.74 .

A confirmation of saturation or escalation cannot of course be obtained from a low order expansion in $J^{\prime 2}$, but even the 'perturbative' coefficient provides a hint of strong coupling physics. It also suggests a smooth evolution from weak to strong coupling.

\section{Double exchange: $J^{\prime} / W \rightarrow \infty$}

In the double exchange limit the $J^{\prime}$ scale acts as a 'constraint' on the electron spin orientation and no longer directly affects physical properties, the only effect is to renormalise the chemical potential. The mapping of the $J^{\prime} / t \rightarrow \infty$ problem to a 'spinless fermion' problem with hopping dependent on nearest neighbour spin orientation has been widely discussed ${ }^{39}$. Transformed to spinless fermions, which correspond to original electron states with spin projection 'locked' parellel to the local quantisation axis, $\mathbf{S}_{i}$, the Hamiltonian becomes:

$$
H=\sum_{\langle i j\rangle} t_{i j} \gamma_{i}^{\dagger} \gamma_{j}=\sum_{\langle i j\rangle} \bar{t} \gamma_{i}^{\dagger} \gamma_{j}+\sum_{\langle i j\rangle} \delta t_{i j} \gamma_{i}^{\dagger} \gamma_{j}
$$

The $t_{i j}$ being the spin orientation dependent hopping amplitude specified earlier. We can split it into the mean (uniform) hopping amplitude, $\bar{t}$, and the fluctuation $\delta t_{i j}$.

In the 'extreme' paramagnetic phase of this model, the distribution of hopping integrals is exactly known. The spins are independently distributed on a sphere so the $t_{i j}$ can be worked out. There is no obvious small parameter, since both the mean value of hopping, $\bar{t}$, as well as the fluctuation, $\Delta t=\sqrt{\left\langle\delta t^{2}\right\rangle}$, are $\propto t$. However, the ratio $\Delta t / \bar{t} \approx 1 / 3$. Numerical work by Li et al. ${ }^{24}$ had demonstrated that less than $0.3 \%$ of states in the band are localised under this condition. It was not clear whether the resistivity at the band center, $n=0.5$, could be described within a Boltzmann approach. Narimanov and Varma ${ }^{40}$ have demonstrated that the mean free path emerging from the Boltzmann calculation is $l / a_{0} \gtrsim 8$ so the method is self-consistent.

It seems now that despite the localisation effects as $n \rightarrow 1$ and $n \rightarrow 0$, resistivity over much of the band can be understood within a effective 'weak coupling' approach. The resistivity is $\approx(0.1-0.2) \rho_{\text {Mott }}$ at the band center according to our calculation. The resistivity is also 
'particle-hole' symmetric, now within the lower band, but notice that this is cleanly visible only at very large $J^{\prime} / t$.

\section{Virtual orbital mixing: large finite $J^{\prime}$}

As we move to finite $J^{\prime}$ from the double exchange limit, the two bands still remain split (down to $J^{\prime} / t \approx 5$ ) but there is a virtual admixture that is introduced. To access properties in this regime we need to use a two orbital formulation, with the orbital energies still separated by a large gap $\sim J^{\prime}$. The chemical potential remains in the lower band. The two orbital model, written in terms of electronic states with local quantisation axis, has the form:

$$
H=\sum_{i j} t_{i j}^{\alpha \beta} \gamma_{i \alpha}^{\dagger} \gamma_{j \beta}-\mu \sum_{i} n_{i}-\frac{J^{\prime}}{2} \sum_{i}\left(n_{i \alpha}-n_{i \beta}\right)
$$

We have not seen a Boltzmann calculation of transport in this regime, but using the two orbital formulation it might be possible to set up such a scheme. The resistivity decreases as we move down from large $J^{\prime}$, so using the correct 'basis' the transport may be accessible within a Boltzmann approach (since the double exchange limit is itself so accessible). The increase in conductivity, $\delta \sigma\left(J^{\prime}\right)$, as we move to lower $J^{\prime}$, is found to be proportional to $1 / J^{\prime}$. A perturbative correction to the large $J^{\prime}$ result, within a diagrammatic scheme yields the same answer.

\section{Behaviour near band tails}

Spin disorder by itself cannot localise states in the center of the band, since $\Delta t / t$ is not large enough. However at the band tails, i.e, $n$ close to 1 or 0 , the kinetic energy is small and a small fraction of states can still be localised. As we have indicated earlier, this is $\lesssim 0.3 \%^{24}$ of the total number of states for $J^{\prime} \rightarrow \infty$.

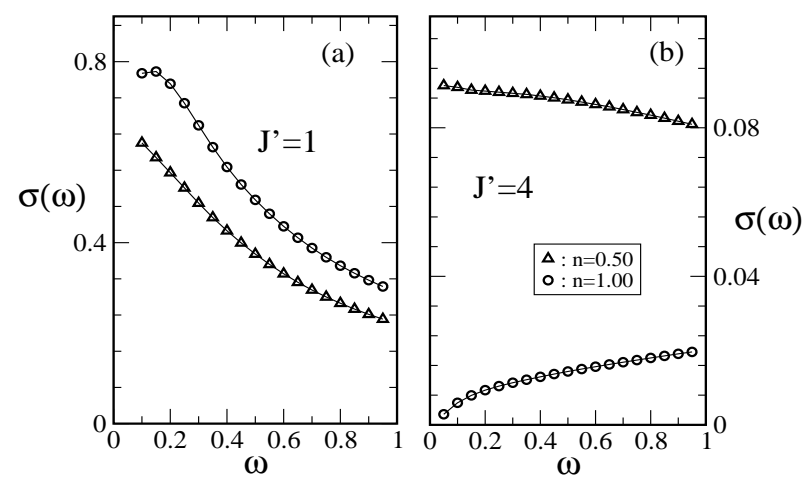

FIG. 12. Optical conductivity: $(a)$. Drude response at both $n=0.5$ and $n=1.0$ at weak coupling, and (b). strong scattering at $n=0.5$ and alomst 'insulating' response at $n=1.0$ at strong coupling.
We do not know if any analytic approaches have been explored in this localisation problem. This regime would be relevant to the low doping magnetic semiconductors, where there is also the possibility of carriers trapping into spin polaronic states.

\section{Optical conductivity}

The optical conductivity confirms the trends seen in the d.c conductivity. Fig. 12 shows $\sigma(\omega)$ at $J^{\prime}=1$ and $J^{\prime}=4$, weak and 'strong' coupling respectively, at the center $(n=0.5)$ and edge $(n=1)$ of the lower band.

At weak coupling, over the frequency range shown, $\sigma(\omega)$ is larger at $n=1$, the center of the full band, compared to $n=0.5$. The scattering rate is $\Gamma_{s}=2 \pi N\left(\epsilon_{F}\right) J^{\prime 2}$ which at the band center is $\approx 0.75$. If $\sigma(\omega)$ follows the Drude form then $\sigma\left(\Gamma_{s}\right) / \sigma(0)$ should be $\sim 0.5$, which is consistent with Fig.12.(a). By $J^{\prime}=4$, the trend has reversed. The $n=1$ case is almost insulating, with $\sigma(0) \rightarrow 0$, while the conductivity at the (lower) band center is finite and essentially flat on the scale considered. This trend gets amplified as we go to even larger $J^{\prime}$.

\section{COMBINED STRUCTURAL AND MAGNETIC DISORDER}

\section{A. Global features}

In the presence of both structural and magnetic disorder it is not possible to show the full density dependence of transport properties compactly, so we provide two generic 'cross sections' in Fig.13 at $n=0.26$ and $n=1.00$. In addition to the effects already noted for potential scattering and magnetic scattering, there are several novel features that arise.

(a). For weak $J^{\prime}$ and moderately large $\Delta$, magnetic scattering weakens localisation effects, as evident from the intermediate $\Delta$ small $J^{\prime}$ data in Fig.13.

(b). At even larger $\Delta$, where the system would have been Anderson localised, magnetic scattering converts the insulator to a metal. The critical disorder $\Delta_{c}(n)$ shifts to a larger value $\Delta_{c}\left(n, J^{\prime}\right)$, see phase diagrams in Fig.14.

(c). In contrast to purely magnetic scattering, where the resistivity typically 'saturates' with increasing $J^{\prime}$, in the presence of structural disorder the system can go insulating with increasing $J^{\prime}$.

$(d)$. The 'additivity' of magnetic and structural scattering holds only over a very limited range in $\Delta$ and $J^{\prime}$, Mathiessens rule generally does not hold.

The major features, above, can be easily motivated after we write down the different effective models of scattering in the various transport regimes in the problem. Some of this has been discussed earlier by us $^{27}$, so we will discuss mainly those aspects of the problem which have not been covered earlier. 


\section{B. Transport regimes}

The parameter space of the problem is large, involving $n-\Delta-J^{\prime}$, and it is convenient to first identify distinct density ranges and then classify the transport/scattering mechanisms. The roughly distinct density regimes are the following.

(1). The wide 'mid band' region $0.1 \lesssim n \lesssim 0.9$, of which $n=0.26$ in Fig.13.(a) is typical, and we have discussed the $n=0.5$ case earlier ${ }^{27}$.

(2). $n \rightarrow 1$, where the response is similar to the mid band region at weak $J^{\prime}$, but the large $J^{\prime}$ response is distinctive, Fig.13.(b).

(3). $n \rightarrow 0$, where structural disorder and magnetic scattering readily leads to localisation.

\section{Generic density: the mid band region}

Let us consider this typical density regime first. For generic densities, $0.1 \lesssim n \lesssim 0.9$, say, there are tentatively five different transport regimes in the problem. These are: $(i)$ Both $\Delta$ and $J^{\prime}$ small: the weak scattering regime, where the effect of structural disorder and magnetic scattering are perturbative and additive. (ii) Moderate $\Delta$ and small $J^{\prime}$ : spin flip correction to weak localisation. The $\Delta$ dependence shows WL corrections and spin flip scattering weakens the WL correction. (iii) Large $\Delta$, $\sim \Delta_{c}$, and small $J^{\prime}$ : spin dephasing driven insulatormetal transition (IMT). (iv) $J^{\prime} / t \rightarrow \infty$, with varying $\Delta$ : the disordered double exchange (DE) limit. $(v) J^{\prime} / t \gg 1$ but finite, and moderate to large $\Delta$ : the intermediate coupling 'metal'.

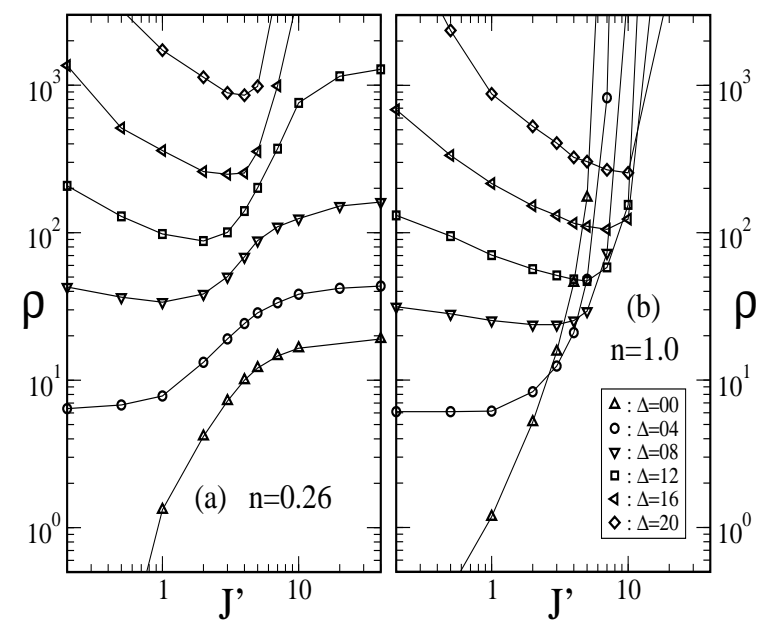

FIG. 13. Global behaviour of the resistivity with varying structural disorder $(\Delta)$ and magnetic coupling $\left(J^{\prime}\right)$ : (a). $n=0.26$ typical of most of band, and (b). $n=1.0$ which, at large $J^{\prime}$, corresponds to the upper edge of the lower band and has its own distinct transport response.
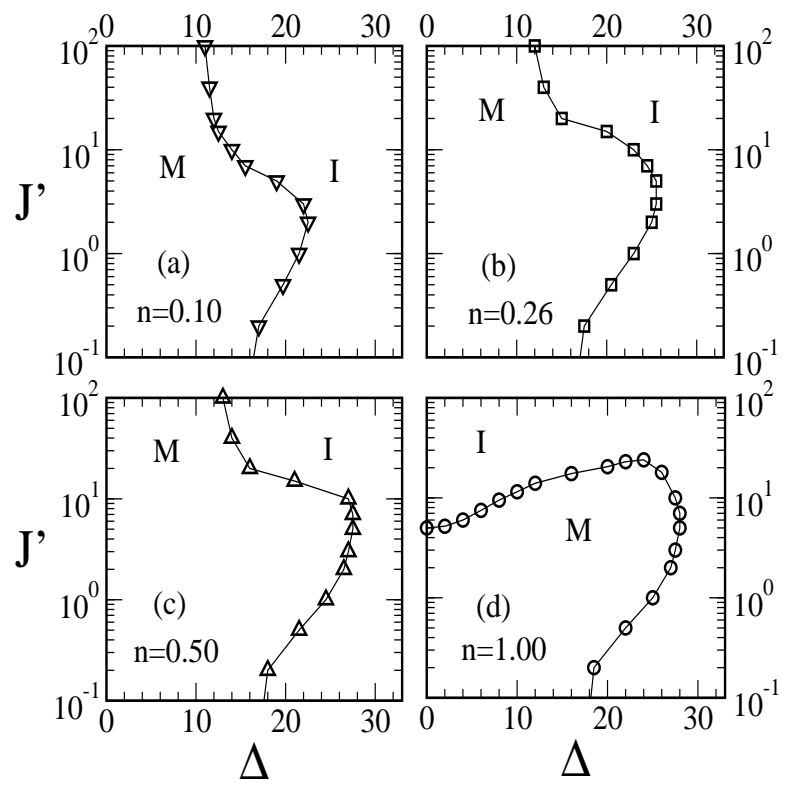

FIG. 14. Putting together different constant density cross-sections to create a 'global' insulator-metal phase diagram for the $\Delta+J^{\prime}$ problem. The densities are marked in the panels. M stands for a metallic phase while I is insulating. The bounding curves can be viewed as $\Delta_{c}\left(n, J^{\prime}\right)$. Notice the $\log$ scale on $J^{\prime}$.

(i) When $\Delta$ and $J^{\prime}$ are both small the transport can be understood in terms of additive Born scattering, with the net scattering rate, $\Gamma\left(\Delta, J^{\prime}\right) \approx a_{1} \Delta^{2}+b_{1} J^{\prime 2}$, and the resistivity $\rho \propto \Gamma\left(\Delta, J^{\prime}\right)$. The 'window' describing this regime is roughly $J^{\prime} \leq 3$ and $\Delta \lesssim 4$. The resistivity in this regime is $\rho<0.1 \rho_{\text {Mott }}$, i.e, below $100 \mu \Omega \mathrm{cm}$, say. This corresponds to the bottom left hand corner in Fig.13.(a) and, as our earlier data showed ${ }^{27}$, Mathiessens rule holds.

(ii) At larger $\Delta$ remaining at small $J^{\prime}$, as the WL corrections show up, spin flip scattering ${ }^{22,23}$ of the electrons by the random magnetic moments reduces the localising effect of structural disorder, i.e, $\partial \rho /\left.\partial J^{\prime}\right|_{n, \Delta}<0$. Just as inelastic scattering weakens quantum interference by introducing decoherence, spin flip scattering leads to spin decoherence. We have quantified the $\Delta$ and $J^{\prime}$ dependence of the effect in the earlier paper ${ }^{27}$.

(iii) At even larger disorder, $\Delta \gtrsim \Delta_{c}$, where the $J^{\prime}=$ 0 system would have been Anderson localised, spin flip scattering opens up a metallic window. The structural disorder needed for localisation shifts to a larger value, i.e, $\partial \Delta_{c} /\left.\partial J^{\prime}\right|_{n}>0$. This effect in visible in all the panels in Fig.14.

(iv) Now consider the DE limit, $J^{\prime} \rightarrow \infty$. As we have discussed in Section IV, the form of the resistivity $\rho\left(J^{\prime}, \Delta=0\right)$ arising from 'magnetic disorder' at large $J^{\prime}$ is very different from what one observes in $\rho\left(J^{\prime}=0, \Delta\right)$ at large $\Delta$. This is because $J^{\prime}$ contributes to both 'band splitting' and effective disorder, and the effective disorder saturates as $J^{\prime} / W \rightarrow \infty$ with $J^{\prime}$ controlling only the 
band splitting. The presence of structural disorder in the $J^{\prime} \rightarrow \infty$ problem strongly enhances the resistivity and localising tendency. Using the transformations used in the previous section:

$$
\begin{aligned}
H & =\sum_{i j} t_{i j}(\theta, \phi) \gamma_{i}^{\dagger} \gamma_{j}+\sum_{i} \epsilon_{i} \gamma_{i}^{\dagger} \gamma_{i} \\
& \equiv \sum_{i j} t_{0} \gamma_{i}^{\dagger} \gamma_{j}+\sum_{i j} \delta t_{i j} \gamma_{i}^{\dagger} \gamma_{j}+\sum_{i} \epsilon_{i} \gamma_{i}^{\dagger} \gamma_{i}
\end{aligned}
$$

The localisation properties of this model have been studied by $\mathrm{Li}$ et $a .^{24}$, although they did not calculate the resistivity. The 'hopping disorder' by itself localises less than $0.5 \%$ of the states in the band. On adding structural disorder the mobility edge moves inward, with localisation of the full band occuring at $\Delta / t \sim 11.5$, which can be approximately understood from the roughly $30 \%$ band narrowing due to spin disorder.

$(v)$ Finally the regime with large but finite $J^{\prime}$ and strong structural disorder. We have seen that the effect of small $J^{\prime}$ at strong structural disorder can be qualitatively understood in terms of the spin dephasing effect on Anderson localisation. However, the small $J^{\prime}$ behaviour with $\partial \rho / \partial J^{\prime}<0$ quickly leads to a minimum and then a regime with $\partial \rho / \partial J^{\prime}>0$. Such behaviour can be viewed as an extension of the $J^{\prime 2}$ term seen at weak disorder, but it is more fruitful to approach the effect from the strong coupling DE end, as we do below. Transforming to the usual local spin quantisation frame and retaining both the parallel and anti-parallel electron states, we have:

$$
H=\sum_{i j} t_{i j}^{\alpha \beta} \gamma_{i \alpha}^{\dagger} \gamma_{j \beta}+\sum_{i} \epsilon_{i} n_{i}-\frac{J^{\prime}}{2} \sum_{i}\left(n_{i \alpha}-n_{i \beta}\right)
$$

The major source of disorder is still $\epsilon_{i}$, with additional contribution from the $\delta t_{i j}^{\alpha \beta}$. The orbital mixing effect of 'off diagonal' couplings, either in terms of mean amplitude or fluctuations, is regulated by the large energy denominator $J$ '. Although the 'reference' problem, $J^{\prime} \rightarrow \infty$, is not analytically tractable in the presence of structural disorder, it can be shown that orbital mixing generates a correction to conductivity $\sim \mathcal{O}\left(1 / J^{\prime}\right)$.

\section{Half-filling: $n \rightarrow 1$}

For $n \rightarrow 1$, the effects at small $J^{\prime}$ are similar to $(i)-$ (iii) at generic densities, discussed above. This is borne out by the behaviour of $\rho\left(J^{\prime}, \Delta\right)$ in Fig.13.(b) and the phase diagram in Fig.14.(d). At large $J^{\prime}$, however, the system always goes insulating, see Fig.13.(b), as we have discussed in Section IV as well. This effect is obviously due to the band splitting induced by large $J^{\prime}$ and the vanishing $\operatorname{DOS}, N\left(\epsilon_{F}\right)$, at $n=1.0$. Thus, for $n=1.0$, the metallic phase is bounded both in $\Delta$ and $J^{\prime}$, Fig.14.(d).

There is however an interesting and possibly unexpected feature in Fig.14.(d) for $J \gtrsim 5.0$, where the $\Delta=0$ system becomes insulating. We may have imagined that introducing structural disorder in this system would enhance localisation. This however is not true, and structural disorder actually 'metallises' the reference band split state, and the critical $J^{\prime}$ needed for localisation increases in the presence of structural disorder.

The origin of the effect above lies in the 'band broadening' effect of structural disorder. The $\Delta=0$ problem had a narrow (vanishingly small) gap in the DOS, and the presence of structural disorder creates finite DOS at the Fermi level, effectively closing the gap. Since the net disorder arising from the random spins and the structural disorder is relatively weak the finite DOS seems sufficient to lead to a metallic, albeit highly resistive, phase. For $\Delta \sim 4$ and $J^{\prime} \sim 5$, the resistivity is roughly $0.5 \rho_{\text {Mott }}$. As $\Delta$ becomes large, or $J^{\prime}$ becomes large, this metallic window is lost due to the effects either of $\Delta$ driven localisation or band splitting.

\section{Very low density: $n \rightarrow 0$}

The case of $n \rightarrow 0$, for example $n=0.01$, say, is unfortunately hard to access with control for the system sizes that we have used. We expect that the small $J^{\prime}$ behaviour will be similar to that in the rest of the band, with enhanced resistivity (due to the low carrier density) while the behaviour for $J^{\prime} \rightarrow \infty$ will be similar to that for $n \rightarrow 1$ (due to the particle-hole symmetry within the lower band, $0<n<1)$. Localisation in the $n \rightarrow 0$ limit, we believe, is better explored via transfer matrix methods due to the large accessible size.

\section{CONCLUDING REMARKS}

In this paper we have presented controlled results on electron transport in the background of arbitrary structural and spin disorder and provided a framework within which the data can be analysed. We benchmarked our Kubo formula based method in the standard problem of potential scattering and Anderson localisation. We then explored the distinct transport regimes that arise in the case of pure magnetic scattering, as well as the combined effect of structural and magnetic disorder. In contrast to the effect of only structural disorder (where the resistivity 'escalates' with increasing disorder) or only magnetic scattering (where it 'saturates' with increasing disorder) their combined action can lead to non monotonic dependence and novel transport regimes. The method developed in this paper can be directly taken over in calculating the resistivity in the presence of annealed disorder, where accessible system sizes rarely exceed $\sim 10^{3}$, and has been extensively used by us in Monte Carlo studies of several "disordered" electron systems.

We acknowledge use of the Beowulf cluster at HRI. S.K. gratefully acknowledges support by the Deutsche Forschungsgemeinschaft through SFB484. 


\section{APPENDIX: EXTRAPOLATION FOR THE D.C CONDUCTIVITY}

It is known that at sufficiently low frequency the optical conductivity in the $3 \mathrm{D}$ Anderson model follows a simple power law ${ }^{19}$ with the exponent depending on the strength of disorder and electron density. This means that we can write: $\sigma(\omega)=A+B \omega^{\alpha}$, where the coefficient $A \geq 0$ is $\sigma_{d c}$, while the next term gives the leading low frequency correction. This form captures all the broad regimes in 3D. For example, at half-filling: (i) low disorder, Born scattering, $\Delta \ll \Delta_{c}$, gives $B<0$, $\alpha=2$, (ii) moderate disorder, weak localisation corrections: $B>0, \alpha=1 / 2,($ iii $)$ critical disorder, $\Delta=\Delta_{c}$ : $A=0, B>0, \alpha=1 / 3$, and (iv) localised phase, $\Delta>\Delta_{c}$ : $A=0, B>0, \alpha=2$.

The form for $\sigma(\omega)$ fixes the form for $\sigma_{a v}(\Delta \omega, L)$. Setting $\Delta \omega \sim 1 / L$, and using the form for $\sigma(\omega)$ above, we obtain the three parameter form for $\sigma_{a v}(L): \sigma_{a v}(L) \sim$ $A+\frac{B}{(\alpha+1)} L^{-\alpha}$. The extrapolation is a least square three parameter fit to our $L$ dependent data, and has enough flexibility to cover all disorder regimes.

In practice, a simpler two parameter extrapolation also works reasonably as long as one is in the metallic phase, even close to the metal-insulator transition: $\sigma_{a v}(L) \sim$ $A+\frac{B}{(3 / 2)} L^{-1 / 2}$ This derives from $\sigma(\omega) \sim A+B \omega^{1 / 2}$. Using this restricted fitting function, the MIT can be roughly located when $A$, the d.c conductivity, falls below a preset limit, $10^{-6}$ say, (which in absolute units is a resistivity $\left.\sim 10^{4} \rho_{M o t t}\right)$. Having located the transition approximately, the more elaborate three parameter fit can be used to confirm the metallic/insulating character on two sides of the critical point. Our MI phase diagrams are constructed using this strategy.

${ }^{1}$ P. A. Lee and T. V. Ramakrishnan, Rev. Mod. Phys. 57, 287 (1985).

${ }^{2}$ B. Kramer and A. MacKinnon, Rep. Prog. Phys. 56, 1469 (1993).

${ }^{3}$ N. F. Mott and E. A. Davis, Electronic Processes in Non Crystalline Materials (Clarendon Press, Oxford, 1979).

${ }^{4}$ N. F. Mott, Metal-Insulator Transitions (Taylor \& Francis, London, 1990)

${ }^{5}$ A. C. Hewson, Kondo Problem to Heavy Fermions (Cambridge University Press, Cambridge, 1993).

${ }^{6}$ Y. Kuramoto and Y. Kitaoka, Dynamics of Heavy Electrons (Oxford University Press, Oxford, 2000).

${ }^{7}$ See, for example, S. Sullow, G. J. Nieuwenhuys, A. A. Menovsky, J. A. Mydosh, S. A. M. Mentink T. E. Mason, and W. J. L. Buyers, Phys. Rev. Lett. 78, 354 (1997).

8 A. Amato, Rev. Mod. Phys. 69, 1119 (1997).

${ }^{9}$ T. Dietl, H. Ohno, F. Matsukura, J. Cibert and D. Ferrand, Science, 287, 1019 (2000).
${ }^{10}$ G. Alvarez, M. Mayr and E. Dagotto, Phys. Rev. Lett, 89, 277202 (2002).

${ }^{11}$ M. Berciu and R.N. Bhatt, Phys. Rev. Lett. 87, 1072 (2001).

12 S. Paschen, D. Pushin, M. Schlatter, P. Vonlanthen, H. R. Ott, D. P. Young and Z. Fisk, Phys. Rev. B 61, 4174 (2000).

${ }^{13}$ S. Sullow, I. Prasad, M. C. Aronson, J. L. Sarrao, Z. Fisk, D. Hristova, A. H. Lacerda, M. F. Hundley, A. Vigliante and D. Gibbs, Phys. Rev. B 57, 5860 (1998).

${ }^{14}$ See, e.g, A. P. Ramirez, J. Phys. Condens Matter 9, 8171 (1997), E. Dagotto, T. Hotta and A. Moreo, Phys Rep 344, 1 (2001), Colossal Magnetoresistive Oxides, edited by Y. Tokura (Gordon \& Breach, 2000).

${ }^{15}$ F. Hellman, M. Q. Tran, A. E. Gebala, E. M. Wilcox and R. C. Dynes, Phys. Rev. Lett. 77, 4652 (1996).

${ }^{16}$ P. Majumdar and S. Kumar, Phys. Rev. Lett. 90, 237202 (2003).

17 S. Legvold in Ferromagnetic Materials, Vol I, edited by E. P. Wohlfarth (North Holland, Amsterdam, 1999).

${ }^{18}$ L. P. Gorkov, A. I. Larkin and D. E. Khmelnitskii, JETP Lett. 30, 248 (1979).

${ }^{19}$ See D. Vollhardt and P. Wolfle in Electronic Phase Transitions, edited by W. Hanke and Yu. V. Kopaev (North Holland, Amsterdam, 1992).

${ }^{20}$ C. M. Soukoulis, E. N. Economou and G. S. Grest, Phys. Rev. B 36, 8649 (1987).

${ }^{21}$ K. Efetov, Supersymmetry in Disorder and Chaos (Cambridge University Press, Cambridge, 1999).

${ }^{22}$ P. A. Lee, J. Non Cryst. Solids, 35, 21 (1980).

${ }^{23}$ S. Hikami, A. I. Larkin and Y. Nagaoka, Prog. Theor. Phys. 63, 707 (1980).

${ }^{24}$ Q. Li, J. Zang, A.R. Bishop and C.M. Soukoulis, Phys. Rev B 56, 4541 (1997).

${ }^{25}$ P. G. de Gennes and J. Friedel, J. Phys. Chem. Solids 4, 71 (1958).

${ }^{26}$ Michael E. Fisher and J. S. Langer, Phys. Rev. Lett. 20, 665 (1968).

${ }^{27}$ Sanjeev Kumar and Pinaki Majumdar, Europhys. Lett. 65, 75 (2004).

${ }^{28}$ See, for example, P. Fazekas, Lecture Notes on Electron Correlation and Magnetism (World Scientific, Singapore, 1999).

${ }^{29}$ Sanjeev Kumar and Pinaki Majumdar, cond-mat 0305345.

${ }^{30}$ Sanjeev Kumar and Pinaki Majumdar, Phys. Rev. Lett. 91, 246602 (2003).

${ }^{31}$ J. Garca Soldevilla, J. C. Gomez Sal, J. A. Blanco, J. I. Espeso, and J. Rodriguez Fernandez, Phys. Rev. B 61, 6821 (2000).

${ }^{32}$ G. D. Mahan, Quantum Many Particle Physics (Plenum Press, New York, 1990).

${ }^{33}$ S. Datta, Electronic Transport in Mesoscopic Systems (Cambridge University Press, Cambridge, 1992).

34 B. K. Nikolic and P. B. Allen, Phys. Rev. B 63, 020201-(R) (2000)

${ }^{35}$ K. Slevin, T. Ohtsuki and T. Kawarabayashi, Phys. Rev. Lett. 84, 3915 (2000).

${ }^{36}$ E. N. Economou, C. M. Soukoulis, and A. D. Zdetsis, Phys. Rev. B 30, 1686 (1984)

37 T. R. Kirkpatrick and D. Belitz, Phys. Rev. B 34, 2168 
(1986).

38 A. Weisse, Eur. Phys. J. B 40, 125 (2004).

${ }^{39}$ E. Muller-Hartmann and E. Dagotto, Phys. Rev. B 54, R6819 (1996).

${ }^{40}$ E. E. Narimanov and C. M. Varma, Phys. Rev. B 65 , 024429 (2002).

${ }^{41}$ D. Yoshioka et al., J. Phys. Soc. Jpn, 50, 3419 (1981). 